\title{
关于 Columbus 问题大扰动情形的完全解
}

\author{
徐 硕 昌 \\ (中国科学院力学研究所, 北京)
}

摘 要

本文在大扰动普遍情形下，按照连续系统的 Ляпунов 直接方法解答了 Columbus 问题. 所得理论结果和 Kelvin 实验给果精确一致. 至此, Columbus 问题得到较完 善的解决。

\section{一、引 言}

Columbus 问题是一个经典问题. 文献 [1] 个绍了它的历史. 文献 [2-4]分别用“一一次近 似变分直接方法”和“Ляпунов 直接方法”按线性理论解答了 Columbus 问题; 文献 [5]给出了 旋转充液腔体非线性稳定问题的普遍数学提法, 并在弱非线性情形下, 按、Пягуннов 方法解答 了 Columbus 问题,在这些结果中,理论和 Kelvin 实验结果都是一致的. 对旋转充液腔体的稳 定问题，文献 [6-8]从不同方面进行了研究.

本文以卡尔丹角为方位参量，给出了轴对称充液腔体自由呬旋的大扰动稳定问题的普遍 数学提法. 这就克服了以 9 个方向余弦为经量带来的困难,使问题提得更精确.

本文是在大扰动情形下，按照连续系统的 Ляпунов 稳定理论解答 Columbus 问题，斦得 结果和 Kelvin 实验精确一致. 文章最后还概述了 Columbus 问题的实际应用.

\section{二、轴对称充液腔体大扰动稳定问题的普遍数学提法}

Columbus 问题的理论模型是研究轴对称充液腔休绕铅亚的对称轴自由迴旋状态的稳定 性. 本文记号和三个坐标系的引人类似文献[5]. 不同处是选择卡尔丹 $\alpha, \beta, \gamma$ 为方位参量.

(1) 固定坐标系 $\{o, \xi\}$ : 坐标原点 $o$ 取在重心上, $\xi_{3}$ 轴铅直朝上,坐标单位基矢为 $i_{1}^{0}, i_{2}^{0}$, $i_{3}^{0}$.

(2) 旋转坐标系 $\{o, x\}: x_{3}$ 轴和 $\xi_{3}$ 轴重合, 此系相对 $\{o, \xi\}$ 系以 $\boldsymbol{\Omega}_{0}=\left(0,0, Q_{0}\right)$ 㭚转, $\boldsymbol{\Omega}$ 为腔体整体旋转的角速度. 坐标单位基矢为 $\boldsymbol{i}_{1}, \boldsymbol{i}_{2}, \boldsymbol{i}_{3}$.

（3）固连坐标系 $\left\{0, x^{\prime}\right\}$. 此系和充液腔体相固连，坐标基矢 $\boldsymbol{i}_{1}^{\prime}, \boldsymbol{i}_{2}^{\prime}, \boldsymbol{i}_{3}^{\prime}$. 沿其惯性 : : 方 向.

设想充液院体被安置正一个卡尔丹环架上,外环 $R_{1}$ 的轴为 $x_{1}$ 轴， $\alpha$ 为绕 $x_{1}$ 轴的转角; 内 㳅 $R_{2}$ 支承于外环 $R_{1}$ 中, 内环 $R_{2}$ 的轴垂直于 $x_{1}$ 轴, $\beta$ 为绕内环轴的转角; 内环 $R_{2}$ 的喓在初始

本文 1984 年 3 月 6 日收到。 


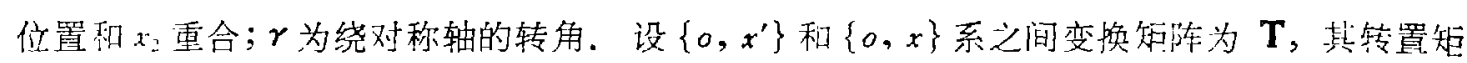
阵 $\mathbf{T}^{*}$. 用 $\alpha, \beta, \gamma$ 角表示方向余弦, 得到 ${ }^{[91}$

$$
\mathbf{T}=\left(\begin{array}{ccc}
\cos \beta \cos \gamma & \cos \alpha \sin \gamma+\sin \alpha \sin \beta \cos \gamma & \sin \alpha \sin \gamma-\cos \alpha \sin \beta \cos \gamma \\
-\cos \beta \sin \gamma & \cos \alpha \cos \gamma-\sin \alpha \sin \beta \sin \gamma & \sin \alpha \cos \gamma+\cos \alpha \sin \beta \sin \gamma \\
\sin \beta & -\sin \alpha \cos \beta & \cos \alpha \cos \beta
\end{array}\right) .
$$

我们选取壳体最大外直径 $d_{\max }$ 为特征长度, $\frac{1}{\Omega_{0}}$ 为特征时间 $T, \rho d^{3} \mathrm{wax}_{\mathrm{x}}$ 为特征质量 $(\rho$ 为液 体密度), 用“〜”记有量纲物理量, 则决定系统运动的无量纲量具有如下形式:

$$
\begin{aligned}
& t=\tilde{t} Q_{0}, \quad L=\frac{\tilde{L}}{d_{\mathrm{max}}}, \quad M=\frac{\widetilde{M}}{\rho d_{\mathrm{max}}^{3}}, \quad p=\frac{\tilde{p}}{\rho d_{\mathrm{max}}^{2} Q_{0}^{2}} \\
& g=\frac{\tilde{g}}{d_{\mathrm{m} x \mathrm{x}} \Omega_{0}^{2}} .
\end{aligned}
$$

以下的物理量和方程均按无量纲形式写出.

腔体内液体平衡时满足:

$$
p^{0}=p_{0}+\frac{1}{2}\left(x_{1}^{2}+x_{2}^{2}\right)-g x_{3},
$$

其中 $p^{0}$ 为无量纲淮作压力, $g$ 为无量纲重力加速度. 在以后记号均表示无量纲量, 不再加以说 明. 下酉相对 $\{0, x\}$ 系列出基本方程和边界条件.

\section{1. 壳体的运动方程}

设壳体和腔内液体转动惯量分量分别为 $A_{1}=B_{1}, C_{1}$ 和 $A_{2}=B_{2}, C_{3}$.

设扰动角速度为 $\boldsymbol{\omega}$, 则壳体的总动量矩为

$$
\boldsymbol{G}=\left(0,0, c_{1}\right)+\mathbf{i}_{3} \cdot \mathbf{J}+\boldsymbol{\omega} \cdot \mathbf{J},
$$

这里 $\mathbf{J}$ 为壳体的惯量张量

$$
\begin{aligned}
\mathbf{J}=T^{*}\left(\begin{array}{ccc}
A_{1} & 0 & 0 \\
0 & A_{1} & 0 \\
0 & 0 & C_{1}
\end{array}\right) \cdot \mathbf{T} \\
=\left(\begin{array}{ccc}
A_{1}+\left(C_{1}-A_{1}\right) \sin ^{2} \beta & \left(A_{1}-C_{1}\right) \sin \alpha \sin \beta \cos \beta & \left(C_{1}-A_{1}\right) \cos \alpha \cos \beta \sin \beta \\
\left(A_{1}-C_{1}\right) \sin \alpha \sin \beta \cos \beta & A_{1}+\left(C_{1}-A_{1}\right) \sin ^{2} \alpha \sin ^{2} \beta & \left(A_{1}-C_{1}\right) \sin \alpha \cos \alpha \cos ^{2} \beta \\
\left(C_{1}-A_{1}\right) \cos \alpha \cos \beta \sin \beta & \left(A_{1}-C_{1}\right) \sin \alpha \cos \alpha \cos ^{2} \beta & A_{1}+\left(C_{1}-A_{1}\right) \cos ^{2} \alpha \cos ^{2} \beta
\end{array}\right) .
\end{aligned}
$$

志体的总动量矩方程为

$$
\begin{aligned}
& \frac{d}{d t}[\boldsymbol{\omega} \cdot \mathbf{J}]+\left\{\boldsymbol{i}_{3} \times[\boldsymbol{\omega} \cdot \mathbf{J}]+\frac{d}{d t}\left[\boldsymbol{i}_{3} \cdot \mathbf{J}\right]\right\}=-\iint_{s} \boldsymbol{r} \times\left(-p_{1} \mathbf{I}+\boldsymbol{\tau}\right) \cdot \boldsymbol{n} d s \\
& -\left(C_{1}+C_{2}-A_{1}-A_{2}\right)\left\{\cos \alpha \sin \alpha \cos ^{2} \beta, \cos \alpha \cos \beta \sin \beta, 0\right\},
\end{aligned}
$$

其中 $p_{1}=p-p^{0}=p+g x_{3}-\frac{1}{2}\left(x_{1}^{2}+x_{2}^{2}\right)-p_{0}$ 一扰动压力,

I 为单位张量, $d s$ 为面积元, $\boldsymbol{s}$ 为啵体比的界面, $\boldsymbol{n}$ 为其外法问单位矢量, $\boldsymbol{\tau}$ 为粘滞应力张 量, $\tau_{i j}=\frac{1}{R_{e}}\left(\frac{\partial v_{i}}{\partial x_{j}}+\frac{\partial \nu_{i}}{\partial x_{i}}\right)$, 
$k_{r}=\frac{e^{d i m} x_{0}}{\mu}$ 为雷诺数.

\section{2. 腔内液体运动方程}

3. 卡尔丹角方程

$$
\begin{aligned}
& \frac{d \boldsymbol{v}}{d t}+2 \boldsymbol{i}_{3} \times \boldsymbol{v}=\nabla\left(-p_{1} \mathbf{I}+\boldsymbol{\tau}\right), \\
& \nabla \cdot \boldsymbol{v}=0 .
\end{aligned}
$$

$$
\begin{aligned}
& \dot{\alpha}=\omega_{1}-\operatorname{tg} \beta\left(\omega_{3} \cos \alpha-\omega_{2} \sin \alpha\right), \\
& \dot{\beta}=\omega_{3} \sin \alpha+\omega_{2} \cos \alpha, \\
& \dot{\gamma} \cos \beta=\omega_{3} \cos \alpha-\omega_{2} \sin \alpha .
\end{aligned}
$$

\section{4. 边界条件}

$$
\left.\boldsymbol{v}\right|_{s}=\boldsymbol{\omega} \times \boldsymbol{r} .
$$

整个非线性稳定问题就是按非线性微分一积分方程组 (5)-(10) 和边界条件 (11) 以及初 始条件求解 $\omega_{1}, \omega_{2}, \omega_{3}, v_{1}, v_{2}, v_{3}, p_{1}, \alpha, \beta, \gamma$. 在整个问题中, 可先由 (5)-(9) 式解出除 $r$ 外的全部未知函数,再由 (10) 式求解 $r$.

\section{三、大扰动稳定理论}

\section{1. 能量积分关系式}

为了导出能量积分关系式,利用如下关系式:

(1) 在 (5) 式等号左端第二项和第三项满足 ${ }^{[8]}$ :

$$
\boldsymbol{i}_{3} \times[\boldsymbol{\omega} \cdot \mathbf{J}]+\frac{d}{d t}\left[\boldsymbol{i}_{3} \cdot \mathbf{J}\right]=\omega \times \boldsymbol{a},
$$

其中 $\boldsymbol{a}=\left[2 J_{13}, 2 J_{23}, J_{33}-J_{11}-J_{22}\right]$

(2) 由方程 (8), (9) 导出如下关系

$$
\begin{aligned}
& \frac{1}{2} \frac{d}{d t}\left(\sin ^{2} \alpha+\cos ^{2} \alpha \sin ^{2} \beta\right) \\
= & \omega_{1} \cos \alpha \sin \alpha \cos ^{2} \beta+\omega_{2} \cos \alpha \cos \beta \sin \beta .
\end{aligned}
$$

类似文献 [5], 以 $\boldsymbol{\omega}$ 标乘 (5) 式两端, 等号左端应用 (12) 式, 右端应用 (13) 式化简; 再 以 $\boldsymbol{v}$ 标乘 (6) 式两端, 对 $\boldsymbol{\tau}$ 积分, 然后将两式相加就导得能量积分关系式:

$$
\frac{d}{d t}\{E(\alpha, \beta, \omega, \boldsymbol{v})+L(\alpha, \beta)\}=-\Phi(\boldsymbol{v}),
$$

其中

$$
\begin{aligned}
E(\alpha, \beta, \omega, \boldsymbol{v}) & =\frac{1}{2} \boldsymbol{\omega} \cdot \mathbf{J} \cdot \omega+\frac{1}{2} \iiint_{\tau}|\boldsymbol{v}|^{2} d \tau \text { 一扰动动能项, } \\
L(\alpha, \beta) & =\left(C_{1}+C_{2}-A_{1}-A_{2}\right)\left(\sin ^{2} \alpha+\cos ^{2} \alpha \sin ^{2} \beta\right) \\
& =a\left(1-\cos ^{2} \alpha \cos ^{2} \beta\right) \text { 一一扰动势能项, }
\end{aligned}
$$

其中 


$$
\begin{gathered}
a=C_{1}+C_{2}-\mathcal{A}_{1}-A_{2} . \\
\Phi(\boldsymbol{v})=\frac{1}{2} \iiint_{\tau} \frac{1}{R_{e}}\left(\frac{\partial v_{i}}{\partial x_{j}}+\frac{\partial v_{j}}{\partial x_{i}}\right)^{3} d \tau \text { 一一粘滞耗散函数. }
\end{gathered}
$$

\section{2. 两个引理}

引理 1. 粘滞散函数 $\Phi(\boldsymbol{v})=0$ 的充要条件是液体作刚体运动, 在定点运汸情形只能. 作整体旋转运动.

\section{引理 2. 液体粘滞耗散函数 $\Phi(v)$ 是正定的.}

证. 证明耗散函数 $\Phi(\boldsymbol{v})$ 的正定性对应求解变分问题：在附加条件 $\operatorname{div} \boldsymbol{v}=0$ 和 $\left.\boldsymbol{v}\right|_{s}=$ $\boldsymbol{\omega} \times \boldsymbol{r}$ 下求解泛函

$$
\Phi(v)=\frac{1}{R_{e}} \iiint_{\tau}|\nabla v|^{2} d \tau
$$

的极小值.

设 $u=v-\omega \times r$, 这是液体相对壳体的无量纲速度，上述变分问题可以变换为以下等 价的变分问题.

在附加条件 $\operatorname{div} \boldsymbol{u}=0$ 和 $\left.\boldsymbol{u}\right|_{s}=0$ 下, 求泛函

$$
\Phi(\boldsymbol{u})=\Phi(\boldsymbol{v})=\frac{1}{R_{e}} \iiint_{s}|\nabla \boldsymbol{u}|^{2} d \tau
$$

的极小值.

应用 Lagrange 不定乘子法并注意到归一化条件

$$
\iiint_{\mathbf{T}}|\mathbf{u}|^{2} d \tau=1
$$

引人新泛函

$$
\mathscr{L}(\boldsymbol{u})=\Phi(\boldsymbol{u})-\frac{\lambda}{2} \iiint_{\tau}|\boldsymbol{u}|^{2} d \tau-\iiint_{\tau} p \operatorname{div} \boldsymbol{u} d \tau .
$$

对 $\mathscr{L}(\mathbf{u})$ 取变分, 假设 $\left.(\boldsymbol{u}+\delta \boldsymbol{u})\right|_{s}=0$,

$$
\begin{aligned}
& \delta \mathscr{L}=\frac{1}{R_{e}} \iiint_{\tau}\left\{2 \frac{\partial u_{1}}{\partial x_{1}} \frac{\partial}{\partial x_{1}} \delta u_{1}+\left(\frac{\partial u_{2}}{\partial x_{1}}+\frac{\partial u_{1}}{\partial x_{2}}\right) \frac{\partial}{\partial x_{2}} \delta u_{1}+\left(\frac{\partial u_{1}}{\partial x_{3}}+\frac{\partial u_{3}}{\partial x_{1}}\right) \frac{\partial}{\partial x_{3}} \delta u_{1}\right. \\
&+\left(\frac{\partial u_{2}}{\partial x_{1}}+\frac{\partial u_{1}}{\partial x_{2}}\right) \frac{\partial}{\partial x_{1}} \delta u_{2}+2 \frac{\partial u_{2}}{\partial x_{2}} \frac{\partial}{\partial x_{2}} \delta u_{2}+\left(\frac{\partial u_{3}}{\partial x_{2}}+\frac{\partial u_{2}}{\partial x_{3}}\right) \frac{\partial}{\partial x_{3}} \delta u_{2} \\
&\left.+\left(\frac{\partial u_{3}}{\partial x_{1}}+\frac{\partial u_{1}}{\partial x_{3}}\right) \frac{\partial}{\partial x_{1}} \delta u_{3}+\left(\frac{\partial u_{3}}{\partial x_{\tau}}+\frac{\partial u_{2}}{\partial x_{3}}\right) \frac{\partial}{\partial x_{2}} \delta u_{3}+2 \frac{\partial u_{3}}{\partial x_{3}} \frac{\partial}{\partial x_{3}} \delta u_{3}\right\} d \tau \\
&-\lambda \iint_{\tau} \int_{\tau}\left(u_{1} \delta u_{1}+u_{2} \delta u_{2}+u_{3} \delta u_{3}\right) d \tau \\
&-\iiint_{\tau} p \nabla \cdot \delta \mathbf{u} d \tau=0 .
\end{aligned}
$$

利用奥一高定理可将 (22) 式化简为: 


$$
\begin{aligned}
\delta \mathscr{L}= & \iint_{r}\left\{-\lambda \boldsymbol{u}-\frac{1}{R_{e}}\left[\nabla^{2} \boldsymbol{u}+\nabla(\operatorname{div} \boldsymbol{u})\right]+\nabla p\right\} \delta \boldsymbol{u} d \boldsymbol{\tau} \\
& +\iint_{s} \delta \boldsymbol{u} \cdot[-p \mathbf{I}+\boldsymbol{\tau}(\boldsymbol{u})] \cdot \boldsymbol{n} d s=0 .
\end{aligned}
$$

由此得到变分问题相应的本征值问题为

$$
\left.\begin{array}{l}
-\lambda \mathbf{u}=-\nabla p+\frac{1}{R} \nabla \boldsymbol{u}, \\
\operatorname{div} \mathbf{u}=0, \\
\left.\mathbf{u}\right|_{s}=0 .
\end{array}\right\}
$$

反之,由本佂值问题 (24) 出发可导出变分问题（20）. 求耗散函数极小值的变分问题和 求解本征值问题 (24) 是等价的.

本佧值问题 (24) 和文献 [10] 导得的本征值问题 (B4.8) 完全一样. 我们要求的最小本 征值恰是文献[10]所要求的最小耗散系数. 文献 [10] Appendix B 对这个问题作了详细讨论. 这个问题在文献 [11] 中从线性非定常问题的存在性和唯一性普遍提法下作了严格处理. 已 经严格证明：本征值问题（24）一定存在最小本征值 $\lambda_{1}$ 和㥵应本征函数 $\boldsymbol{u}_{1}$ 使泛函 $\Phi(\boldsymbol{u})$ 当 $\boldsymbol{u}=\mathbf{u}_{1}$ 时取极小，满足：

$$
\Phi(\boldsymbol{u})=\Phi(\boldsymbol{v}) \geqslant \Phi\left(\boldsymbol{u}_{1}\right)=\frac{\lambda_{1}}{2} \iiint_{\tau}\left|\boldsymbol{u}_{1}\right|^{2} d \tau
$$

至此证明了耗散函数的正定性.

\section{3. 稳定性的定义}

由于方程组 (5)-(9) 中不含 $\gamma$, 只有 (10) 式含 $\gamma$, 因此, 我们先对方程组 (5)-(9) 的 所有可能解组 $\mathscr{U}=\{\alpha, \beta, \omega, \boldsymbol{v}\}$ 构成的泛函空间 $\Psi=\mathscr{R}^{2} * \mathscr{R}^{3} \times C^{2}(\tau)$ 上来讨论解的稳 定性. 这里 $C^{2}(\tau)$ 为在区域 $\tau$ 上有定义, 且满足 $\nabla \cdot \boldsymbol{v}=0$ 和 $\left.\boldsymbol{v}\right|_{s}=\omega \times \boldsymbol{r}$ 的连续可微函数 $\boldsymbol{v}(\boldsymbol{r}, t)$ 的企枿。

平衡解就是零解, 即

$$
\overline{\mathscr{U}}=\{\alpha, \beta, \boldsymbol{\omega}, \boldsymbol{v}\}=\{0,0,0,0\},
$$

充液腔体的平衡解 $\overline{\mathscr{U}}$ 的稳定性按距离

$$
\rho(\mathscr{U}, 0)=\left\{\sin ^{2} \alpha+\sin ^{2} \beta+|\boldsymbol{\omega}|^{2}+\iiint_{\tau}|\boldsymbol{v}|^{2} d \tau\right\}^{\frac{1}{2}}
$$

来定义.

假设 $-\frac{\pi}{2}<\alpha<\frac{\pi}{2},-\frac{\pi}{2}<\beta<\frac{\pi}{2}$, 如果 $\alpha=\beta=\frac{\pi}{2}$ 系统平躺在水平面上就认为不 稳定. 这里按距离 (27) 式定义的稳定性具有明确物理意义. 如果旋转充液腔体平衡解是渐 近稳定的, 则当 $t \rightarrow \infty$ 时, $\alpha \rightarrow 0, \beta \rightarrow 0, \omega \rightarrow 0, \iiint_{\tau}|\boldsymbol{v}|^{3} d \tau \rightarrow 0$, 再由 (10) 式得 $\dot{\gamma} \rightarrow 0$. 这表明充分长时问后, 充液腔体就仍回到未扰动前的状态, 初态和终态只有 $r$ 为常数的一个位 相差,对于轴对称体这是无须区别的. 


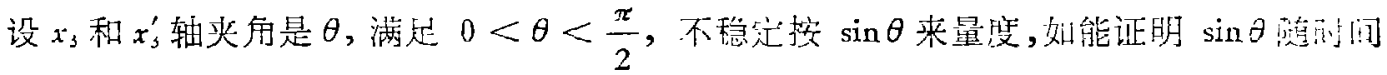
$\boldsymbol{t}$ 单调增加, 表明系统偏离铅垂方向越来越大, 不稳定包含的物理意义是很朋确的.

\section{4. 稳定性判据}

定理 1. 如果轴对称充液腔体绕铅垂对称轴的自由迴旋(重心为定点)状态的唅能具有极 小值，即

$$
L(\alpha, \beta)=a\left(\sin ^{2} \alpha+\cos ^{2} \alpha \sin ^{2} \beta\right)>0,
$$

则对于满足条件 $-\frac{\pi}{2}<\alpha<\frac{\pi}{2}$ 和 $-\frac{\pi}{2}<\beta<\frac{\pi}{2}$ 的任意大扰劯, 系统的自由聥泟惊态都 是渐近稳定的.

证. 由 (4) 式可得如下估计

$$
|\boldsymbol{\omega}|^{2} \min \left(A_{1}, C_{1}\right) \leqslant \boldsymbol{\omega} \cdot \mathbf{J} \cdot \boldsymbol{\omega}^{*} \leqslant|\boldsymbol{\omega}|^{2} \max \left(A_{1}, C_{1}\right) .
$$

势能表达式 $(28)$ 可变换为

$$
L(\alpha, \beta)=\frac{a}{4}\left[(3+\cos 2 \beta) \sin ^{2} \alpha+(3+\cos 2 \alpha) \sin ^{2} ; 3\right] .
$$

作 Ляпунов 泛函为

$$
\begin{aligned}
V(\mathscr{U})= & \frac{a}{4}\left[(3+\cos 2 \beta) \sin ^{2} \alpha+\left(3+\cos 2(x) \sin ^{2} \beta\right]\right. \\
& +\boldsymbol{\omega} \cdot \mathbf{J} \cdot \boldsymbol{\omega}+\frac{1}{2} \iiint_{\tau}|\boldsymbol{v}|^{2} d \tau .
\end{aligned}
$$

容易验证 $V(\mathscr{U})$ 关于 $\rho(\mathscr{U}, 0)$ 是正定的, 即

$$
\alpha_{1} \rho^{2}(\mathbb{R}, 0) \leqslant V(\mathscr{U}) \leqslant \alpha_{2} \rho^{2}(\mathbb{U} \ell, 0),
$$

其中

$$
\begin{aligned}
& \alpha_{1}=\min \left(\frac{a}{2}, A_{1}, C_{1}, \frac{1}{2}\right), \\
& \alpha_{2}=\max \left(a, f_{1}, C_{1}, \frac{1}{2}\right),
\end{aligned}
$$

仅当 $\mathscr{Q}=0, V(\mathscr{Q} U)=V(0)=0$.

根据能量积分关系式 (14) 和引理 2 的 (25) 式得到

$$
\frac{d V(\mathscr{U})}{d t}=-\Phi(v)=-\Phi(\boldsymbol{u}) \leqslant-\frac{\lambda_{1}}{2} \iiint_{\tau}\left|\boldsymbol{u}_{1}\right|^{2} d \tau,
$$

即 $\frac{d V(\mathscr{U})}{d t}$ 是负定的.

根据 $V(\mathscr{Q} \ell)$ 的上述性质证得平衡关于 $\rho(\mathscr{Q} \ell, 0)$ 是渐近稳定的. 系统当 $t \rightarrow \infty$ 时, $\alpha \rightarrow$ $0, \beta \rightarrow 0|\omega| \rightarrow 0$, 和 $\iiint_{\tau}|\boldsymbol{v}|^{2} d \tau \rightarrow 0$, 再由 $(10)$ 式得 $\dot{\gamma} \rightarrow 0$. 系统和未扰动前平衡状态只 存在 $\boldsymbol{r}$ 为常数的相位差.

定理 2. 如果轴对称充液腔体绕铅垂的对称轴自由迥旋状态势能取极大值,即

$$
L(\alpha, \beta)=a\left(\sin ^{2} \alpha+\cos ^{2} \alpha \sin ^{2} \beta\right)<0,
$$




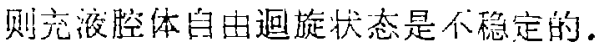

证. 设对称轴和铅垂方向夹角为 $\theta$, 则势能可变换为:

$$
L(\alpha, \beta)=a\left(1-\cos ^{2} \theta\right)=a \sin ^{2} \theta .
$$

由能量积分关系式 (14) 对 $t$ 积分得：

$$
E(\alpha, \beta, \omega, v)+L(\theta)=-\int_{t_{0}}^{t} \phi(\boldsymbol{v}) d t+E\left(\alpha_{0}, \beta_{0}, \omega_{0}, \boldsymbol{v}_{0}\right)+L\left(\theta_{0}\right),
$$

其中 $E\left(\alpha_{0}, \beta_{0}, \omega_{0}, v_{0}\right)$ 为初始时刻 $\left(t=t_{0}\right)$ 的扰动动能, $L\left(\theta_{0}\right)$ 为初始扰动势能.

只要假设初始扰动满足条件

$$
E\left(\alpha_{0}, \beta_{0}, \omega_{0}, v_{0}\right)<\left|L\left(\theta_{0}\right)\right| .
$$

利用 (39) 式和引理 2 的 (25) 式, (38) 式变换得到:

$$
\begin{aligned}
|a| \sin ^{2} \theta & >\int_{t_{0}}^{t} \Phi(v) d t+\left|E\left(\alpha_{0}, \beta_{0}, \omega_{0}, \boldsymbol{v}_{0}\right)+L\left(\theta_{0}\right)\right| \\
& >\frac{\lambda_{0}}{2}\left(t-t_{0}\right) \iiint_{\tau}\left|\boldsymbol{u}_{1}\right|^{2} d \tau+\left|E\left(\alpha_{0}, \beta_{0}, \omega_{0}, \boldsymbol{v}_{0}\right)+L\left(\theta_{0}\right)\right| .
\end{aligned}
$$

由 (40) 式看出, 对称轴偏离铅垂方向 ( $\theta$ 角)随时间 $t$ 单调增加,定理 2 得证.

综合定理 1 和定理 2 得到:

定理 3. 轴对称充液腔体绕铅垂的对称轴自由迴旋运动稳定的充要条件是势能具有极小 值, 即

$$
C_{1}+C_{2}>A_{1}+A_{2} \text {. }
$$

推论 1. 当轴对称充液腔体是扁形 $(C>A)$ 则, 则其绕铅亚的对称轴自由迥旋是渐近稳 定的; 如米它是长形的 $(C<A)$ 则是不稳定的.

\section{四、Columbus 问题的应用}

利用上节推论 1 解答 Columbus 问题是一自了然的. Kelvin 流体转子陀蝶仪实验结果扁 形转子是稳定的; 长形转子是不稳定的, 这和推论 1 的结论精确地一致. 至此, 我们从大扰动 普遍情形出发按照 Ляпунов 直接方法给出了 Columbus 问题的完善解答.

Columbus 问题的应用包括

1. 工程技术方面: 本文在大扰动普遍情形建立了流体转子陀螺仪的稳定准则: 转子必须设 计成府形。这对于陀螺仪设计、液体然料火箭、各类飞行器的设计均有一定理论指导意义 ${ }^{12}{ }^{14}$.

2. 天体演化学方面：为重新肯定 “Jeans-Darwin 理论”提供强有力的实验证据 ${ }^{[13-15]}$.

3. 地球物理学方面: 从埋论和实验两方面证明了牛顿地球模型的自转运动是稳定的 ${ }^{1]}$.

\section{参考文献}

[1] 稌硕昌,自然控志, 5(1982), 1:22-24.

[2]-—, 力学学报, 1981 特刊, 31-36. 
[3] 一, 中国科学, $1999,9: 857-865$.

[4] - - 科学通报, 26(1981), 1:14-17.

[5] - 中国科学, 1982 3:254-264.

[0] 李 每, 应用数学和力学, 4(1983), 5:609.

[i] 一, 应用数学和力学, 4(1983) 6 期.

[8]秦元勋、管克英、李雨，科学通报，29(1984)，4:198-201.

[9] Magnus, K., 陀螺理论与应用(贾书惠等㶦), 国防工业出版社, 1983.

[10] Joseph, D. D., Stability of Fluid Motion, Springer-Verleg, 1976.

[11] Лодытенская, О. А., 粘性不可压缩流动力学的数学问题(张开明译)，上海科吱出版社，1963

「12] 徐顿昌, 力学进展, 1983, 2:146-162.

13] 一, 中国科学 $1981,7: 665-674$.

114] - - 才学进展, 1983, 3:320-327.

[15] 一, 自然杂志, 6(1983)，10:770-7/2. 